\title{
Two-Description Image Coding With Steganography
}

\author{
Zhiyuan Zhang, Ce Zhu, Senior Member, IEEE, and Yao Zhao, Member, IEEE
}

\begin{abstract}
For two-description image coding, a conventional scheme is to partition an image into two parts and then to produce each description by alternatively concatenating a finely coded bitstream of one part and a coarsely coded bitstream of the other part. This letter presents a new two-description image coding approach using steganography. Specifically, we propose forming each description by embedding (hiding) the coarsely coded part into the finely coded part based on a least-significant bit (LSB) steganographic method. In this way, the bit budget for the coarsely coded part in each description can be saved with little reconstruction degradation for the finely coded part if the embedding process is well designed. The experimental results substantiate the effectiveness of the proposed method.
\end{abstract}

Index Terms-Data embedding, image coding, multiple description coding, steganography.

\section{INTRODUCTION}

$\mathbf{R}$ ECENTLY, multiple description (MD) coding has emerged as an attractive framework for robust transmission over unreliable networks. It can efficiently combat packet loss without any retransmission, thus satisfying the demand of real-time services and relieving the network congestion [1]. In MD coding, two or more bitstreams called descriptions of the same image are generated, which can be independently decoded. At the same time, the descriptions should carry correlated information (redundancy). The correlated information is beneficial in the case of single-description reception, in that it helps the estimation of the missing description from the received one. A minimum fidelity in the reconstruction can be obtained at the receiver when only one channel works. When more channels work, a higher fidelity reconstruction can be yielded by combining the descriptions. However, an extra rate is necessary to encode correlated information, which impairs the rate-distortion performance. In this letter, two-description coding is considered as in most papers and applications.

Lots of MD coding techniques have been developed using different strategies for coding various data like speech, audio, image, and video. One of the most classical methods is multiple description scalar quantization (MDSQ) [2], which was successfully applied in image coding [3]. The pairwise correlating transform (PCT) was exploited in [4]. MD lattice vector quantization (MDLVQ) has also shown promising results for image coding [5]. Another popular two-description scheme par-

Manuscript received July 01, 2008; revised August 27, 2008. This work was supported in part by National Natural Science Foundation of China (No. 60776794, No. 90604032), 973 program (No. 2006CB303104), 863 program (No. 2007AA01Z175), PCSIRT (No. IRT0707), and Specialized Research Foundation of BJTU. The associate editor coordinating the review of this manuscript and approving it for publication was Dr. Nikolaos V. Boulgouris.

Z. Zhang and Y. Zhao are with the Institute of Information Science, Beijing Jiaotong University, Beijing 100044, China (e-mail: fractalzzy@126.com; yzhao@center.bjtu.edu.cn).

C. Zhu is with School of Electrical and Electronic Engineering, Nanyang Technological University, 639798 Singapore (e-mail: eczhu@ntu.edu.sg).

Digital Object Identifier 10.1109/LSP.2008.2006710

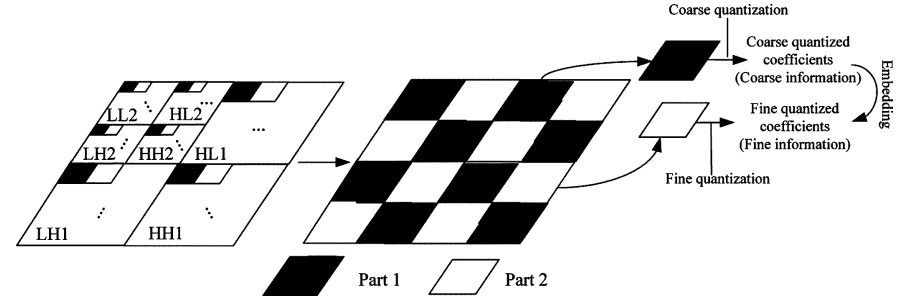

Fig. 1. Basic idea of the two-description image coding with steganography.

titions an (transformed) image into two parts, e.g., part 1 and part 2, each of which goes through a fine coding as well as a coarse coding. Then one description is formed by concatenating the finely coded part 1 and the coarsely coded part 2 , while the other description is complementarily generated by combining the coarsely coded part 1 with the finely coded part 2 . Such MD coding schemes can be found in [6]-[8].

In this letter, we propose a novel two-description image coding scheme. As usual, each description of an image is constructed by a finely coded part (fine information) and another coarsely coded part (coarse information). The new scheme features that the coarse information is embedded (hidden) into the fine information selectively using, for example, an LSB steganographic method [9]. In this way, coarse information can be carried on the fine information freely without allocating any more bit budget for the coarse information. A method combining MD coding and data hiding scheme was proposed in [10], where the DC value is replicated and embedded into the available AC coefficients in both descriptions for DCT-based two-description coding. Compared with that simple method, our scheme is different in the MD coding design and embedding approach. More details for our design will be elaborated in the following sections.

The rest of this letter is organized as follows. In Section II, the basic idea is formulated and the related techniques are described. In Section III, the design method of the proposed two-description coding with steganography is presented. Experimental results are given in Section IV. We conclude this letter in Section V.

\section{BASIC IDEA AND RELATED TECHNIQUES}

Consider partitioning an image into two parts in the block checkerboard manner, where the partition can be done in the spatial or wavelet domain. A block partition in the spatial domain is simple, while the block partition in the wavelet domain normally produces better coding performance, where the wavelet coefficients in different subbands corresponding to the same spatial location are grouped to form tree-structured blocks. Fig. 1 shows such a block checkerboard partition in the wavelet domain.

\section{A. Fine and Coarse Coding}

Two sub-images are grouped by part 1 and part 2 blocks in Fig. 1, respectively. The wavelet coefficients of part 1 sub-image 


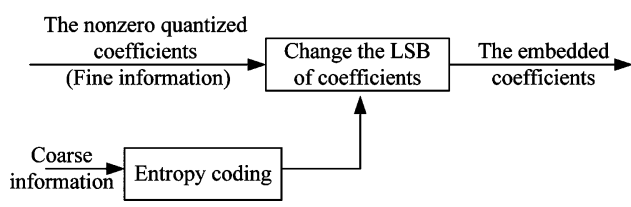

Fig. 2. LSB steganographic method.

are quantized by a coarse quantizer and the quantized coefficients are referred to be coarse information. The wavelet coefficients of part 2 are quantized by a fine quantizer to produce fine information. The coarse information is embedded (hidden) into the fine information. Note that the length of the coarse information (denoted as $L$ ) needs to be transmitted for extracting purpose and the bit cost for the length is trivial and negligible. For example, for a $512 \times 512$ image with 0.1 bpp coarse information, the bit cost for the length representation is about $\log _{2}\left(0.1 \times 512^{2}\right) \doteq 15$ bits, which amounts to an overhead of $15 /(512 \times 512)=5.9 \times 10^{-5}$ bit-per-pixel (bpp).

\section{B. Embedding Approach-LSB Steganographic Method}

Steganography can send message under the cover of a carrier signal, and many techniques of steganography have been proposed [9], [11]. One of the well-known steganographic methods is the LSB substitution which replaces the least-significant bits of coefficients with the information bits. In this letter, the LSB steganographic method is exploited to embed the coarse information in the least-significant bits of the fine information selectively. The process of the LSB steganographic method is shown in Fig. 2.

The principle of the LSB steganographic method is to change the LSB of nonzero quantized wavelet coefficients to match the coarse information bitstream in the following way:

$$
e_{-} w_{i}= \begin{cases}\widetilde{w_{i}}, & \text { if } \bmod \left(\widetilde{w_{i}}, 2\right)=c_{j} \\ \widetilde{w_{i}}+1, & \text { if } \bmod \left(\widetilde{w_{i}}, 2\right) \neq c_{j} \& \bmod \left(\widetilde{w_{i}}, 2\right)=1 \\ \widetilde{w_{i}}-1, & \text { if } \bmod \left(\widetilde{w_{i}}, 2\right) \neq c_{j} \& \bmod \left(\widetilde{w_{i}}, 2\right)=0\end{cases}
$$

where $e_{-} w_{i}$ is the embedded wavelet coefficient, $\widetilde{w_{i}}$ is the original nonzero quantized wavelet coefficient, $\bmod ($,$) is the$ module operation, and $c_{j}, j=1, \ldots, L$ is the $j$ th bit in the coarse information bitstream. by

The information extracting is simple, which can be obtained

$$
c_{j}=\bmod \left(e_{-} w_{i}, 2\right) .
$$

\section{Proposed Two-Description Image Coding Scheme}

The proposed two-description image coding scheme based on wavelet domain partition is sketched in Fig. 3, including encoding and decoding processes. A similar coding structure can be obtained if spatial partitioning is considered.

\section{A. Information Embedding}

The coarse coefficients (coarse information) are embedded into the nonzero quantized wavelet coefficients selected from high frequency to the low frequency for minimizing the degradation of the fine coefficients with the LSB steganographic method introduced in Section II-B, if L is smaller than the number of the nonzero quantized wavelet coefficients. There

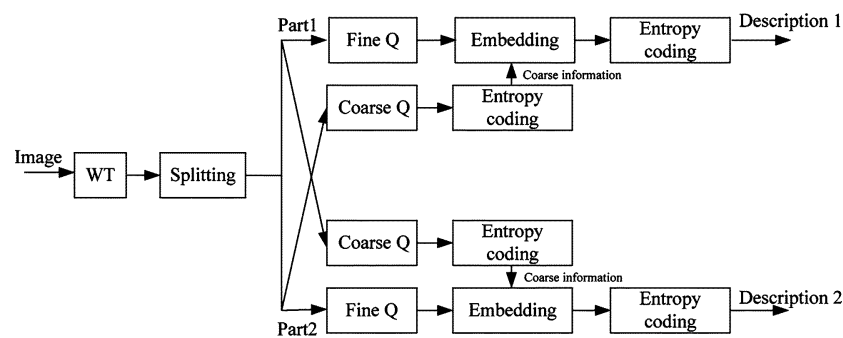

(a)

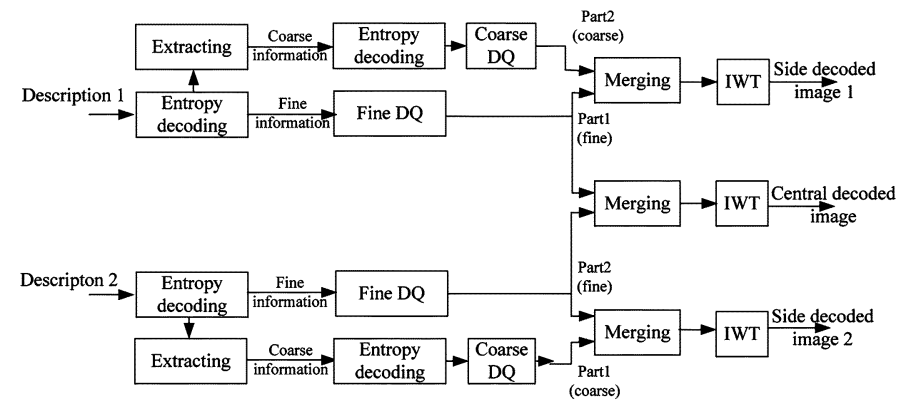

(b)

Fig. 3. Proposed two-description wavelet image coding. (a) Encoding. (b) Decoding.

are two points which need to be considered in the embedding process.

1) Embedding Capacity: The embedding capacity depends on the number of nonzero quantized wavelet coefficients. When the coarse information amount is larger than the embedded capacity, a combination of embedding and appending the coarse information can be employed. That means some coarse information is embedded in the fine information while the rest will be appended after the fine information.

2) Embedding Performance: The embedding of coarse information suggests that the coarse information can be delivered freely (with zero bit rate), which can enhance the side decoding performance when only one description is correctly received. On the other hand, the embedding will affect the quality of the fine information decoding, which may compromise the central decoding performance for two descriptions received correctly. Note that the LSB embedding method expressed in (1) may not necessarily change the fine information, in view that the LSB of the coefficient $\widetilde{w_{i}}$ only changes when $\bmod \left(\widetilde{w_{i}}, 2\right) \neq c_{j}$, which accounts for around $50 \%$ possibility. In this sense, the "free" coarse information can be obtained with the embedding scheme at the cost of a possible degradation of fine information. We would point out that the fine information degradation is normally low, in that only the LSB is changed and the wavelet coefficients are selected from high frequency to low frequency. It is rational to infer that the best way can be to calculate the coding gain and loss, respectively, and then to decide whether or not such an embedding of some bits in some selected coefficients should proceed. Reversible data hiding may also be considered for optimization.

We would like to highlight that the embedding of coarse information into the fine information is very different from simply lending one bit from fine information representation to the coarse information representation (that is again the concatenating of fine and coarse information). In our embedding scheme, the encoded coarse information is embedded into finely quantized wavelet coefficients selectively and then the coefficients are entropy coded. In this way, coarse information 
is included while the finely quantized wavelet coefficients are encoded with only a slight degradation, as to be shown in our following experiments. Also as pointed out above, the embedding may not necessarily change the LSB of the finely quantized wavelet coefficients, and thus, some pieces of fine information may not be affected at all while the coarse information is embedded.

In this letter, we consider embedding all the coarse information into the fine information to check the feasibility and effectiveness of the proposed approach. Further extension and optimization mentioned above will be done in the next step.

\section{B. Discriminative Quantization}

Considering that the LSB of the nonzero quantized wavelet coefficients may be changed for information embedding which may lead to an appreciable change if the quantization step is large, a discriminative quantization scheme is exploited which employs a finer quantization for the nonzero quantized coefficients selected for embedding. Firstly, all the wavelet coefficients are quantized by a uniform quantizer with a quantization stepsize of $q_{1}$. Then for those nonzero quantized coefficients chosen for embedding, a smaller quantization stepsize $\left(q_{2}\right)$ is discriminatively applied for the finer quantization of the coefficients sequentially from the highest frequency to the lowest frequency. The number of the finely quantized coefficients depends on the amount of the coarse information to be embedded, which is indicated by the coarse encoder described in Section II-A.

\section{Decoding}

When both descriptions are received, the finely coded information from each description is used for the reconstruction of the central decoded image. When only one description is available, the coarse information can be extracted from the received description, which is then decoded to complement the decoded fine information for a side decoded image.

\section{EXPERIMENTAL RESULTS}

Six standard images $(512 \times 512)$ are used as the testing images and the 10-18 Daubechies wavelet is employed. For the coarse information, the quantization value is chosen from 65 to 210 for different bit rates tested, where all the coarse information can be embedded into the fine information. By adjusting the finer quantization value $q_{1}$ from 8 to 65 , the bit rate per description varies, and $q_{2}$ is selected as 2 for a small modification due to embedding.

For a fair comparison, we tested our proposed embeddingbased coding scheme against the following two conventional methods without such embedding, with the same coarse and fine coders for the two parts. That is to say, the fine information and the coarse information used for the three testing schemes are exactly the same.

1) Coarse Information Skipping: In the coarse information skipping approach, each description only contains the fine information from the fine coder and skips the coarse information, resulting in the same coding rate per description as in our coarse information embedding scheme. In this way, the redundancy between the two descriptions is minimized to favor the central decoding performance. When only one description is received, an interpolation method used in [12] is exploited to recover the missing part.

2) Coarse Information Appending: In the coarse information appending scheme, the coarse information is appended after the fine information. Each description is produced by concatenating the fine information and the coarse information for the two parts alternatively. Note that the coarse information and the fine information used in this scheme are the same as those in the proposed scheme.

Figs. 4 and 5 plot the rate-distortion performance of side/central coding to compare our proposed method against the other two relevant schemes on two testing images, respectively. Both spatial and wavelet domain partitions are considered in the comparison, where $8 \times 8$ blocks are used for the spatial splitting and $32 \times 32$ tree-structured blocks are for the wavelet domain splitting. In the experiment, the bit rate of coarse information ranges from 0.05 to $0.12 \mathrm{bpp}$. As mentioned before, in this letter, we consider all the coarse information to be embedded into the fine information. We take the spatial domain partition as an example for the following discussions. It can be seen from Figs. 4(a) and 5(a) that, for the side coding, the proposed method outperforms the coarse information skipping approach substantially as the bit rate increases, e.g., over $3 \mathrm{~dB}$ gain for "Lena" and 4 $\mathrm{dB}$ gain for "Barbara." Compared with the coarse information appending method, the proposed scheme can achieve the similar decoded quality (PSNR) with a lower bit rate up to $12.9 \%$ rate reduction for "Lena" and $12.6 \%$ rate reduction for "Barbara." For the central coding, the coarse information skipping method achieves the best central decoded results as expected, at the expense of the poorest side decoded results described above. Figs. 4(b) and 5(b) demonstrate that the proposed one achieves similar results (with a PSNR degradation less than $0.6 \%$ and $0.8 \%$ for "Lena" and "Barbara," respectively) as the coarse information skipping method in central coding, and significantly better results than the coarse information appending scheme due to the bit rate saving. From the results, we can clearly see that our proposed scheme achieves overall better coding results than the two conventional methods in terms of side and central distortion-rate performance.

If we compare the results based on the wavelet domain partition, the figures also clearly demonstrate that the proposed scheme significantly outperforms the appending method, where the improvement is more noticeable, especially for side decoded results, than that based on the spatial partition. The skipping scheme produces very poor side coding results due to the difficulty of interpolating the missing part based on the wavelet domain partition, which is far from the proposed and appending schemes and therefore not shown in the figures. As expected, the wavelet domain partition can achieve better coding performance than the spatial partition. In short, our proposed embedding scheme exhibits superior coding efficiency to the other two conventional approaches in either partition case.

To save space due to the page limit, we cannot include more rate-distortion curves but instead tabulate the results for the other four testing images in Table I based on spatial domain partition for comparison, which further show the coding gains of the proposed scheme over the other two conventional methods. The following parameter settings were used in our experiment: $q_{1}=30, q_{2}=2$ for the results in the table.

\section{CONCLUSION}

In this letter, a new idea for designing two-description coding with steganography has been presented. Instead of concatenating the two encoded parts to construct a description, one coarsely coded part is embedded into the other finely coded part using the LSB steganographic method. A specific embedding-based two-description image coding scheme has 


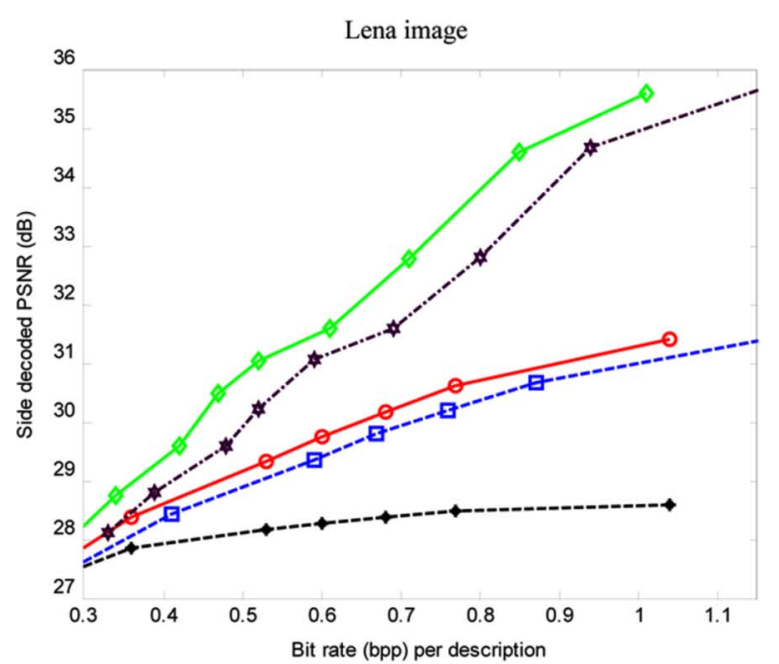

(a)

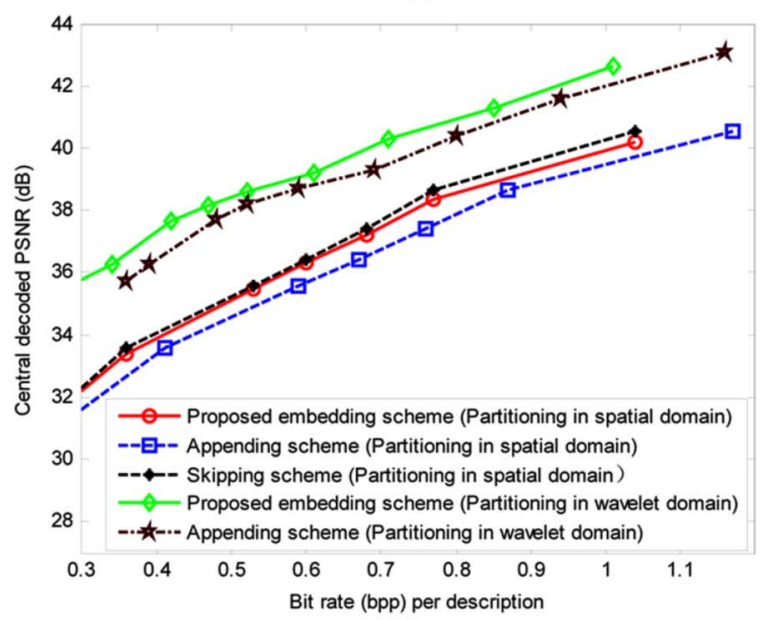

(b)

Fig. 4. Rate-distortion performance comparison for "Lena" image. (a) Side coding. (b) Central coding.

been developed and tested to demonstrate the effectiveness of the proposed scheme with very encouraging results.

\section{REFERENCES}

[1] V. K. Goyal, "Multiple description coding: Compression meets the network," IEEE Signal Process. Mag., vol. 18, no. 5, pp. 74-93, Sep. 2001.

[2] V. Vaishampayan, "Design of multiple description scalar quantizers," IEEE Trans. Inf. Theory, vol. 39, pp. 821-834, 1993.

[3] S. D. Servetto, K. Ramchandran, V. A. Vaishampayan, and K. Nahrstedt, "Multiple description wavelet based image coding," IEEE Trans. Image Process., vol. 9, no. 5, pp. 813-826, May 2000.

[4] Y. Wang, M. Orchard, and A. Reibman, "Multiple description coding using pairwise correlation transforms," IEEE Trans. Image Process., vol. 10, no. 3, pp. 351-366, Mar. 2001.

[5] H. Bai, C. Zhu, and Y. Zhao, "Optimized multiple description lattice vector quantization for wavelet image coding," IEEE Trans. Circuits Syst. Video Technol., vol. 17, pp. 912-917, 2007.

[6] Y. Liu and S. Oraintara, "Feature-oriented multiple description wavelet-based image coding," IEEE Trans. Image Process., vol. 16, pp. 121-131, 2007.

[7] A. Norkin, A. Gotchev, K. Egiazarian, and J. Astola, "Two-stage multiple description image coders: Analysis and comparative study," Signal Process.: Image Commun., vol. 21, pp. 609-625, 2006.

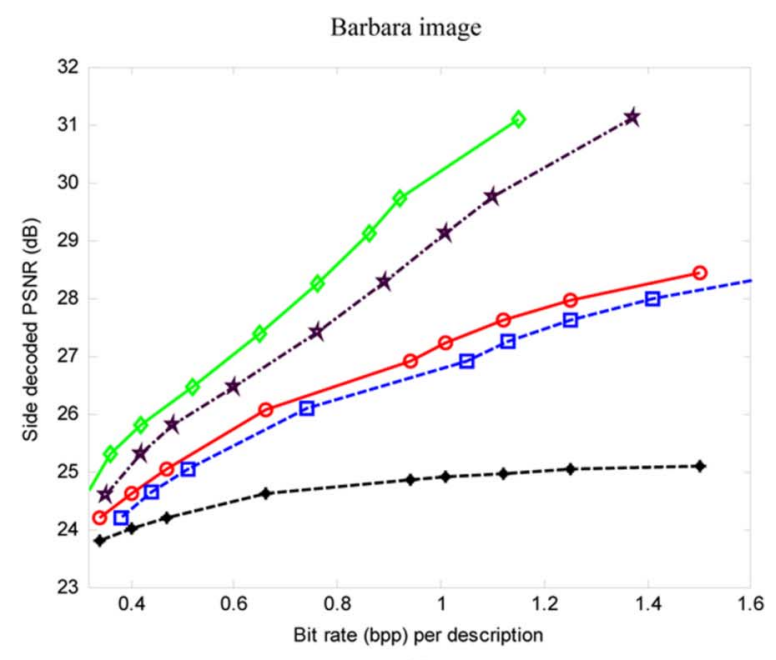

(a)

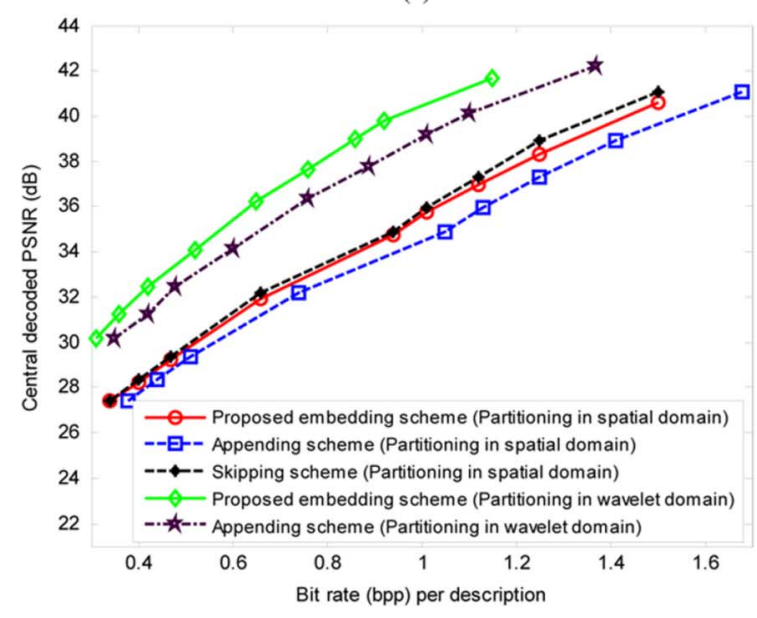

(b)

Fig. 5. Rate-distortion performance comparison for "Barbara" image. (a) Side coding. (b) Central coding.

TABLE I

COMPARISON OF SIDE/CENTRAL CODING RESUlTS FOR THE THREE TESTED SCHEMES

\begin{tabular}{l|lll|ll}
\hline & & Proposed & Skipping & \multicolumn{2}{c}{ Appending } \\
\hline Image & $\begin{array}{l}\text { Rate } \\
\text { (bpp) }\end{array}$ & $\begin{array}{l}\text { Side/Central } \\
\text { PSNR (dB) }\end{array}$ & $\begin{array}{l}\text { Side/Central } \\
\text { PSNR (dB) }\end{array}$ & $\begin{array}{l}\text { Rate } \\
\text { (bpp) }\end{array}$ & $\begin{array}{l}\text { Side/Central } \\
\text { PSNR (dB) }\end{array}$ \\
\hline Pepper & 0.64 & $28.70 / 34.40$ & $28.26 / 34.49$ & 0.71 & $28.70 / 34.49$ \\
Elain & 0.62 & $29.45 / 32.85$ & $29.11 / 32.88$ & 0.68 & $29.45 / 32.88$ \\
Goldhill & 0.76 & $28.70 / 33.06$ & $27.96 / 33.09$ & 0.85 & $28.70 / 33.09$ \\
Girl & 0.62 & $30.43 / 36.99$ & $29.44 / 37.16$ & 0.68 & $30.44 / 37.16$ \\
\hline
\end{tabular}

[8] T. Tillo, M. Grangetto, and G. Olmo, "Multiple description image coding based on Lagrangian rate allocation," IEEE Trans. Image Process., vol. 16, pp. 673-683, 2007.

[9] W. Bender, N. Morimoto, and A. Lu, "Techniques for data hiding," IBM Syst. J., vol. 35, pp. 313-336, 1996.

[10] G. Boato, M. Carli, N. Conci, F. G. B. De Natale, and A. Neri, "Improving perceptual quality of multiple description coding by data hiding," in Proc. 3rd Int. Workshop Video Processing and Quality Metrics for Consumer Electronics, 2007.

[11] D. C. Wu and W. H. Tsai, "A steganography method for images by pixel value differencing," Pattern Recognit. Lett., vol. 24, pp. 1613-1626, 2003.

[12] P. Salama, N. B. Shroff, and E. J. Delp, "Error concealment technique for encoded video streams," in Proc. Int. Conf. Image Processing (ICIP 1995), Oct. 1995, vol. 1, pp. 9-12. 